Commun. Fac. Sci. Univ. Ank. Ser. A1 Math. Stat.

Volume 69, Number 1, Pages 336-346 (2020)

DOI: $10.31801 /$ cfsuasmas.473923

ISSN 1303-5991 E-ISSN 2618-6470

http://communications.science.ankara.edu.tr/index.php?series=A1

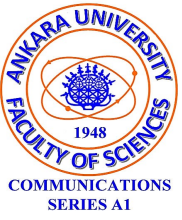

\title{
LIGHTLIKE HYPERSURFACES WITH PLANAR NORMAL SECTIONS IN $\mathbb{R}_{1}^{4}$
}

\author{
FEYZA ESRA ERDOĞAN AND SELCEN YÜKSEL PERKTAS
}

\begin{abstract}
In the present paper our aim is to investigate lightlike hypersurfaces of $\mathbb{R}_{1}^{4}$ having degenerate or non-degenerate planar normal sections. Firstly, we prove that lightlike hypersurfaces in $\mathbb{R}_{1}^{4}$ always have degenerate planar normal sections. Then we examine the conditions for lightlike hypersurfaces in $\mathbb{R}_{1}^{4}$ to have non-degenerate planar normal sections and obtain some characterizations for such lightlike hypersurfaces.
\end{abstract}

\section{INTRODUCTION}

In Euclidean spaces, B.Y. Chen [2] initiated the study of surfaces with planar normal sections. After this, an important literature has been created on such surfaces and submanifolds (for example, see [2, 6], 7, , 9, , 8]). The semi-Riemannian adaptation of such surfaces was done by Y. H. Kim [7]. Recently, the authors ([12], 11]) introduced lightlike surfaces with planar normal sections in Minkowski 3-space and halflightlike submanifolds of $\mathbb{R}_{2}^{4}$ having degenerate and non-degenerate planar normal sections (see also [13]).

By a similar manner in [12 and [11 we define the normal section of a lightlike hypersurface $N$ in $\mathbb{R}_{1}^{4}$ and non-degenerate planar normal sections as follows:

For a point $p$ in a lightlike hypersurface $N$ of $\mathbb{R}_{1}^{4}$ and a lightlike vector $\xi$ such that the radical space $\operatorname{Rad}(T N)=\operatorname{Span}\{\xi\}$, the vector $\xi$ and transversal space $\operatorname{tr}(T N)$ to $N$ at $p$ determine a 2-dimensional subspace $E(p, \xi)$ in $\mathbb{R}_{1}^{4}$ through $p$. The intersection $N \cap E(p, \xi)$ gives rise to a lightlike curve $\alpha$ in a neighborhood of $p$, which we call normal section of $N$ at the point $p$ in the direction of $\xi$. If each normal section $\alpha$ at $p$ in the direction of $\xi$ satisfies $\alpha^{\prime} \wedge \alpha^{\prime \prime} \wedge \alpha^{\prime \prime \prime}=0$, for each $p \in N$, then we say that $N$ has degenerate pointwise planar normal sections.

Received by the editors: October 23, 2018; Accepted: October 07, 2019.

2010 Mathematics Subject Classification. Primary 53C42; Secondary 53C50.

Key words and phrases. Lightlike hypersurface, non-degenerate planar normal section, degenerate normal section.

(C)2020 Ankara University Communications Faculty of Sciences University of Ankara-Series A1 Mathematics and Statistics 
On the other hand, let $w$ be a non-degenerate vector tangent to $\stackrel{N}{N}$ at $p$ such that $w \in S(T N)=S p\{u, v\}$, where $S(T N)$ is the screen distribution of $N$. Then the vector $w$ and transversal space $\operatorname{tr}(T N)$ to $N^{\prime}$ at $p$ determine a 2- dimensional subspace $E(p, w)$ in $\mathbb{R}_{1}^{4}$ through $p$. From the intersection of $N$ and $E(p, w)$, we have a non-degenerate curve $\alpha$ in a neighborhood of $p$ which is called the normal section of $N$ at $p$ in the direction of $w$. In this case, if $\alpha^{\prime} \wedge \alpha^{\prime \prime} \wedge \alpha^{\prime \prime \prime}=0$ is satisfied, for each point $p$ in $N^{\prime}$, where $\alpha$ is a normal section of $N^{\prime}$ at $p$ in the direction of $w$, then $N$ is said to have non-degenerate pointwise planar normal sections.

In this paper, we study lightlike hypersurfaces in $\mathbb{R}_{1}^{4}$ having degenerate and nondegenerate planar normal sections. We prove that every lightlike hypersurfaces of $\mathbb{R}_{1}^{4}$ has degenerate planar normal sections. Also we obtain some results for a lightlike hypersurface with non-degenerate planar normal sections. We prove that a lightlike hypersurface $N$ in $\mathbb{R}_{1}^{4}$ has non-degenerate planar normal sections if and only if it is either screen conformal and totally umbilical or totally geodesic. We also obtain a characterization for non-umbilical screen conformal lightlike hypersurface with non-degenerate planar normal sections.

\section{Preliminaries}

Let $(\breve{N}, \breve{g})$ be an $(n+2)$-dimensional semi-Riemannian manifold with the indefinite metric $\breve{g}$ of index $q \in\{1, \ldots, n+1\}$ and $N$ be a hypersurface of $\breve{N}$. We denote the tangent space at $x \in \hat{N}$ by $T_{x} \hat{N}$. Then

$$
T_{x} \hat{N}^{\perp}=\left\{V_{x} \in T_{x} \breve{N} \mid \breve{g}_{x}\left(V_{x}, W_{x}\right)=0, \forall W_{x} \in T_{x} \hat{N}\right\}
$$

and

$$
\operatorname{Rad} T_{x} N^{\prime}=T_{x} N^{\prime} \cap T_{x} N^{\perp} .
$$

Then, $N$ is called a lightlike hypersurface of $\breve{N}$ if $\operatorname{Rad} T_{x} \hat{N} \neq\{0\}$, for any $x \in N^{\prime}$. Thus $T N^{\perp}=\bigcap_{x \in N^{\prime}} T_{x} N^{\perp}$ becomes a 1- dimensional distribution $\operatorname{RadTN}$ on $N$. Then there exists a vector field $\xi \neq 0$ on $N$ such that

$$
g(\xi, X)=0, \quad \forall X \in \Gamma(T N),
$$

where $g$ is the induced degenerate metric tensor on $N$. We denote the algebra of differential functions on $N$ by $F(N)$ and the $F(N)$-module of differentiable sections of a vector bundle $E$ over $N$ by $\Gamma(E)$.

A complementary vector bundle $S(T N)$ of $T N^{\perp}=\operatorname{Rad} T N^{\prime}$ in $T N^{\prime}$ defined by

$$
T N^{\prime}=\operatorname{RadTN} \oplus_{\text {orth }} S(T N),
$$

is called a screen distribution on $N$. It follows from the equation above that $S(T N)$ is a non-degenerate distribution. Moreover, since we assume that $N$ is para-compact, there always exists a screen $S(T N)$. Thus, along $N$ we have

$$
T \breve{N}_{\mid \hat{N}}=S\left(T N^{\prime}\right) \oplus_{\text {orth }} S\left(T N^{\perp}\right)^{\perp}, \quad S(T N) \cap S\left(T N^{\perp}\right)^{\perp} \neq\{0\},
$$


that is, $S\left(T N^{\perp}\right)^{\perp}$ is the orthogonal complement to $S\left(T N^{\prime}\right)$ in $\left.T \breve{N}\right|_{\hat{N}}$. Note that $S(T N)^{\perp}$ is also a non-degenerate vector bundle of rank 2. However, it includes $T N^{\perp}=\operatorname{Rad} T N^{\prime}$ as its sub-bundle.

Let $(N, g, S(T N))$ be a lightlike hypersurface of a semi-Riemannian manifold $(\breve{N}, \breve{g})$. Then there exists a unique vector bundle $\operatorname{tr}(T \hat{N})$ of $\operatorname{rank} 1$ over $N^{N}$, such that for any non-zero section $\xi$ of $T N^{\perp}$ on a coordinate neighborhood $U \subset N$, there exists a unique section $N$ of $\operatorname{tr}(T N)$ on $U$ satisfying: $T N^{\perp}$ in $S(T N)^{\perp}$ and take $V \in \Gamma\left(\left.F\right|_{U}\right), V \neq 0$. Then $\breve{g}(\xi, V) \neq 0$ on $U$, otherwise $S(T N)^{\perp}$ would be degenerate at a point of $U[5$. Define a vector field

$$
N=\frac{1}{\breve{g}(V, \xi)}\left\{V-\frac{\breve{g}(V, V)}{2 \breve{g}(V, \xi)} \xi\right\},
$$

on $U$ where $V \in \Gamma\left(\left.F\right|_{U}\right)$ such that $\breve{g}(\xi, V) \neq 0$. Then we have

$$
\breve{g}(N, \xi)=1, \breve{g}(N, N)=0, \breve{g}(N, W)=0, \forall W \in \Gamma\left(\left.S(T N)\right|_{U}\right) .
$$

Moreover, from (1) and (2) we have the following decomposition:

$$
\left.T \breve{N}\right|_{\dot{N}}=S(T N) \oplus_{\text {orth }}\left(T N^{\perp} \oplus \operatorname{tr}\left(T N^{\prime}\right)\right)=T N^{\prime} \oplus \operatorname{tr}\left(T N^{\prime}\right) .
$$

Locally, suppose $\{\xi, N\}$ is a pair of sections on $U \subset N$ satisfying (3). Define a symmetric $\digamma(U)$-bi-linear form $B$ and a 1-form $\tau$ on $U$. Hence on $U$, for $X, Y \in$ $\Gamma\left(\left.T N\right|_{U}\right)$, we write

$$
\begin{aligned}
\breve{\nabla}_{X} Y & =\breve{\nabla}_{X} Y+B(X, Y) N, \\
\breve{\nabla}_{X} N & =-A_{N} X+\tau(X) N,
\end{aligned}
$$

which are called local Gauss and Weingarten formula, respectively. Since $\breve{\nabla}$ is a metric connection on $\breve{N}$, it is easy to see that

$$
B(X, \xi)=0, \forall X \in \Gamma\left(\left.T N\right|_{U}\right) .
$$

Consequently, the second fundamental form of $N$ is degenerate [5]. Define a local 1-from $\eta$ by

$$
\eta(X)=\breve{g}(X, N), \forall \in \Gamma\left(\left.T N\right|_{U}\right) .
$$

Let $P$ denote the projection morphism of $\Gamma(T N)$ on $\Gamma(S(T N))$ with respect to the decomposition (1). We obtain

$$
\begin{aligned}
\breve{\nabla}_{X} P Y & =\breve{\nabla}_{X}^{*} P Y+C(X, P Y) \xi \\
\breve{\nabla}_{X} \xi & =-A_{\xi}^{*} X+\varepsilon(X) \xi=-A_{\xi}^{*} X-\tau(X) \xi,
\end{aligned}
$$

where $\breve{\nabla}_{X}^{*} P Y$ and $A_{\xi}^{*} X$ belong to $\Gamma(S(T N)), \breve{\nabla}$ and $\breve{\nabla}^{*}$ are linear connections on $\Gamma\left(S\left(T N^{\prime}\right)\right)$ and $T N^{\perp}$, respectively, $h^{*}$ is a $\Gamma\left(T N^{\perp}\right)$-valued $\digamma\left(N^{\prime}\right)$-bi-linear form on $\Gamma(T N) \times \Gamma\left(S\left(T N^{\prime}\right)\right)$ and $A_{\xi}^{*}$ is $\Gamma\left(S\left(T N^{\prime}\right)\right.$ )-valued $\digamma(N)$-linear operator on $\Gamma\left(T N^{\prime}\right)$. 
We call them the screen fundamental form and screen shape operator of $S(T N)$, respectively. Define

$$
\begin{aligned}
C(X, P Y) & =\breve{g}\left(h^{*}(X, P Y), N\right), \\
\varepsilon(X) & =\breve{g}\left(\breve{\nabla}_{X}^{* t} \xi, N\right), \forall X, Y \in \Gamma(T N) .
\end{aligned}
$$

One can easily show that $\varepsilon(X)=-\tau(X)$. Here, $C(X, P Y)$ is called the local screen fundamental form of $S(T N)$. Precisely, the two local second fundamental forms of $N$ and $S(T N)$ are related to their shape operators by

$$
\begin{aligned}
B(X, Y) & =\breve{g}\left(Y, A_{\xi}^{*} X\right), \\
A_{\xi}^{*} \xi & =0, \\
\breve{g}\left(A_{\xi}^{*} P Y, N\right) & =0 \\
C(X, P Y) & =\breve{g}\left(P Y, A_{N} X\right), \\
\breve{g}\left(N, A_{N} X\right) & =0 .
\end{aligned}
$$

A lightlike hypersurface $(N, g, S(T N))$ of a semi-Riemannian manifold is called totally umbilical[5] if there is a smooth function $\varrho$, such that

$$
B(X, Y)=\varrho g(X, Y), \forall X, Y \in \Gamma(T N),
$$

where $\varrho$ is non-vanishing smooth function on a neighborhood $U$ in $N$.

A lightlike hypersurface $\left(N^{\prime}, g, S\left(T N^{\prime}\right)\right)$ of a semi-Riemannian manifold is called screen locally conformal if the shape operators $A_{N}$ and $A_{\xi}^{*}$ of $N$ and $S(T N)$, respectively, are related by

$$
A_{N}=\varphi A_{\xi}^{*},
$$

where $\varphi$ is non-vanishing smooth function on a neighborhood $U$ in $N$. Therefore, it follows that for any $X, Y \in \Gamma(S(T N))$ and $\xi \in \operatorname{Rad} T N^{\prime}$ we have

$$
C(X, \xi)=0 .
$$

For details about screen conformal lightlike hypersurfaces, we refer [1] and [5] .

\section{Planar Normal Sections of Lightlike Hypersurfaces in $\mathbb{R}_{1}^{4}$}

Let $N$ be a lightlike hypersurface of $\mathbb{R}_{1}^{4}$. Now we shall investigate lightlike hypersurfaces with degenerate planar normal sections. If $\alpha$ is a null curve, for a point $p$ in $N$, we have

$$
\begin{aligned}
\alpha^{\prime}(s) & =\xi \\
\alpha^{\prime \prime}(s) & =\breve{\nabla} \xi \xi=-\tau(\xi) \xi, \\
\alpha^{\prime \prime \prime}(s) & =-\left[\xi(\tau(\xi))+\tau^{2}(\xi)\right] \xi .
\end{aligned}
$$

Then, $\alpha^{\prime \prime \prime}$ is a linear combination of $\alpha^{\prime}$ and $\alpha^{\prime \prime}$. Thus from (21), (22) and (23), we conclude $\alpha^{\prime \prime \prime} \wedge \alpha^{\prime \prime} \wedge \alpha^{\prime}=0$.

Hence we give 
Corollary 1. Every lightlike hypersurface of $\mathbb{R}_{1}^{4}$ has degenerate planar normal sections.

Let $N$ be a lightlike hypersurface of $\mathbb{R}_{1}^{4}$. For a point $p$ in $N$ and a spacelike vector $w \in S\left(T N^{\prime}\right)=S p\{u, v\}$, where $u, v$ are unit spacelike vectors tangent to $N$ at $p$, the vector $w$ and transversal space $\operatorname{tr}(T N)$ to $N$ at $p$ determine a 2dimensional subspace $E(p, w)$ in $\mathbb{R}_{1}^{4}$ through $p$. The intersection of $N$ and $E(p, w)$ gives a spacelike curve $\alpha$ in a neighborhood of $p$, which is called the normal section of $N$ at $p$ in the direction of $w$.

Now, we shall research the conditions for a lightlike hypersurface of $\mathbb{R}_{1}^{4}$ to have non-degenerate planar normal sections.

Let $\left(\hat{N}, g, S\left(T N^{\prime}\right)\right)$ be a totally umbilical and screen conformal lightlike hypersurface of $\left(\mathbb{R}_{1}^{4}, \breve{g}\right)$. In this case $S(T N)$ is integrable [1]. We denote integral hypersurface of $S(T N)$ by $N^{\prime}$. Then, using (6), 11) and $(19)$ we find

$$
\begin{aligned}
C(w, w) \xi+B(w, w) N & =\breve{g}(w, w)\{\rho \xi+\beta N\} \\
& =\lambda\{\rho \xi+\beta N\}, \lambda=a^{2}+b^{2},
\end{aligned}
$$

where $\lambda, \rho, \beta \in \mathbb{R}$. In this case, we obtain

$$
\begin{aligned}
\alpha^{\prime}(s) & =w, \\
\alpha^{\prime \prime}(s) & =\breve{\nabla}_{w}^{*} w+C(w, w) \xi+B(w, w) N, \\
\alpha^{\prime \prime}(s) & =\breve{\nabla}_{w}^{*} w+\rho \xi+\beta N,
\end{aligned}
$$

and

$$
\begin{aligned}
\alpha^{\prime \prime \prime}(s)= & \breve{\nabla}_{w}^{*} \breve{\nabla}_{w}^{*} w+C\left(w, \breve{\nabla}_{w}^{*} w\right) \xi+w(C(w, w)) \xi \\
& -C(w, w) A_{\xi}^{*} w+w(B(w, w)) N \\
& -B(w, w) A_{N} w+B\left(w, \breve{\nabla}_{w}^{*} w\right) N
\end{aligned}
$$

which implies

$$
\begin{aligned}
\alpha^{\prime \prime \prime}(s)= & \breve{\nabla}_{w}^{*} \breve{\nabla}_{w}^{*} w+C\left(w, \breve{\nabla}_{w}^{*} w\right) \xi \\
& +B\left(w, \breve{\nabla}_{w}^{*} w\right) N-\rho A_{\xi}^{*} w-\beta A_{N} w .
\end{aligned}
$$

Here $\breve{\nabla}^{*}$ and $\breve{\nabla}$ are linear connections on $S(T N)$ and $\Gamma(T N)$, respectively and $\alpha^{\prime}(s)=w=a u+b v, a, b \in \mathbb{R}$. Since $N$ is a totally umbilical screen conformal lightlike hypersurface, we find

$$
C\left(w, \breve{\nabla}_{w}^{*} w\right) \xi+B\left(w, \breve{\nabla}_{w}^{*} w\right) N=g\left(w, \breve{\nabla}_{w}^{*} w\right)\left\{\rho_{1} \xi+\beta_{1} N\right\},
$$

where $\rho_{1}, \beta_{1} \in \mathbb{R}$. On the other hand we write

$$
\breve{\nabla}_{w}^{*} w=a^{2} \breve{\nabla}_{u}^{*} u+a b \breve{\nabla}_{u}^{*} v+a b \breve{\nabla}_{v}^{*} u+b^{2} \breve{\nabla}_{v}^{*} v
$$

and

$$
g\left(w, \breve{\nabla}_{w}^{*} w\right)=a^{3} g\left(u, \breve{\nabla}_{u}^{*} u\right)+a^{2} b g\left(u, \breve{\nabla}_{u}^{*} v\right)+a^{2} b g\left(u, \breve{\nabla}_{v}^{*} u\right)+a b^{2} g\left(u, \breve{\nabla}_{v}^{*} v\right)
$$




$$
+a^{2} b g\left(v, \breve{\nabla}_{u}^{*} v\right)+a b^{2} g\left(v, \breve{\nabla}_{u}^{*} v\right)+a b^{2} g\left(v, \breve{\nabla}_{v}^{*} u\right)+b^{3} g\left(v, \breve{\nabla}_{v}^{*} v\right) .
$$

Since $\breve{g}(u, u)=\breve{g}(v, v)=1$ and $\breve{g}(u, v)=0$, then by a direct computation, we obtain

$$
\begin{gathered}
\breve{\nabla}_{u}^{*} u=\lambda_{1} v, \breve{\nabla}_{v}^{*} u=\lambda_{2} v, \\
\lambda_{1}=-\lambda_{3}, \\
\lambda_{2}=-\lambda_{4}, \\
\breve{\nabla}_{u}^{*} v=\lambda_{3} u, \breve{\nabla}_{v}^{*} v=\lambda_{4} u,
\end{gathered}
$$

where $\lambda_{1}, \lambda_{2}, \lambda_{3}, \lambda_{4} \in \mathbb{R}$. Hence, from (32)-35) we get

$$
g\left(w, \breve{\nabla}_{w}^{*} w\right)=0
$$

and

$$
C\left(w, \breve{\nabla}_{w}^{*} w\right) \xi+B\left(w, \breve{\nabla}_{w}^{*} w\right) N=0
$$

Therefore, we obtain

$$
\begin{aligned}
& C\left(w, \breve{\nabla}_{w}^{*} w\right)=0, \\
& B\left(w, \breve{\nabla}_{w}^{*} w\right)=0 .
\end{aligned}
$$

Since $N$ is screen conformal, we find

$$
\begin{aligned}
\alpha^{\prime}(s) & =w \\
\alpha^{\prime \prime}(s) & =\lambda(\rho \xi+\beta N), \\
\alpha^{\prime \prime \prime}(s) & =-\lambda \rho A_{\xi}^{*} w-\lambda \beta A_{N} w,
\end{aligned}
$$

where $\rho, \beta \neq 0$. Then, we have

$$
\alpha^{\prime \prime \prime}(s)=t A_{\xi}^{*} w, t=-2 \lambda \rho .
$$

Hence, we obtain

$$
B(w, w)=g\left(A_{\xi}^{*} w, w\right)=\beta g(w, w)=g(\beta w, w),
$$

which implies $A_{\xi}^{*} w=\beta w$, that is, $\alpha^{\prime}$ and $\alpha^{\prime \prime \prime}$ are linearly dependent and so $N$ has non-degenerate planar normal sections.

Assume that $N$ is a totally geodesic lightlike hypersurface of $\mathbb{R}_{1}^{4}$. Then, we have $B=0, A_{\xi}^{*}=0$. Hence, from (25)- 28), we write

$$
\begin{aligned}
\alpha^{\prime}(s) & =w, \\
\alpha^{\prime \prime}(s) & =\breve{\nabla}_{w}^{*} w, \\
\alpha^{\prime \prime \prime}(s) & =\breve{\nabla}_{w}^{*} \breve{\nabla}_{w}^{*} w .
\end{aligned}
$$

Since $\alpha^{\prime}, \alpha^{\prime \prime}, \alpha^{\prime \prime \prime} \in \Gamma\left(S\left(T N^{\prime}\right)\right)$ and $\operatorname{dim}\left(S\left(T N^{\prime}\right)\right)=2$, we have $\alpha^{\prime \prime \prime}(s) \wedge \alpha^{\prime \prime}(s) \wedge$ $\alpha^{\prime}(s)=0$. 
Conversely, we assume that $N$ has non-degenerate planar normal sections. Then, from 25, 26) and (28) we obtain

$$
w \wedge\left(\begin{array}{c}
\breve{\nabla}_{w}^{*} w+C(w, w) \xi \\
+B(w, w) N
\end{array}\right) \wedge\left(\begin{array}{c}
\breve{\nabla}_{w}^{*} \breve{\nabla}_{w}^{*} w+C\left(w, \breve{\nabla}_{w}^{*} w\right) \xi+w(C(w, w)) \xi \\
-C(w, w) A_{\xi}^{*} w+w(B(w, w)) N \\
-B(w, w) A_{N} w+B\left(w, \breve{\nabla}_{w}^{*} w\right) N
\end{array}\right)=0 .
$$

Since $w=a u+b v, a, b \in \mathbb{R}$, for the sake of simplicity, we choose $u=(0,1,0,0)$ and $v=(0,0,1,0)$, which give

$$
\breve{\nabla}_{w}^{*} w=\left(0, a b \lambda_{3}+b^{2} \lambda_{4}, a^{2} \lambda_{1}+a b \lambda_{2}, 0\right) .
$$

If we take $a=b=1$, from $(32)-(34)$, we obtain

$$
\breve{\nabla}_{w}^{*} w=\left(0,-\left(\lambda_{1}+\lambda_{2}\right), \lambda_{1}+\lambda_{2}, 0\right),
$$

which yields that $w$ and $\breve{\nabla}_{w}^{*} w$ are linearly dependent. Thus we find

$$
w \wedge \breve{\nabla}_{w}^{*} w=0
$$

for any $a, b \in \mathbb{R}$. Moreover, if we take $a, b \in\{-1,1\}$, we have

$$
\breve{\nabla}_{w}^{*} w=\left(0, b\left(a \lambda_{1}+b \lambda_{2}\right), a\left(a \lambda_{1}+b \lambda_{2}\right), 0\right),
$$

namely, in any case $w$ and $\breve{\nabla}_{w}^{*} w$ are linearly dependent.

From (31), we find

$$
\begin{aligned}
\breve{\nabla}_{w}^{*} \breve{\nabla}_{w}^{*} w= & a^{3} \lambda_{1} \lambda_{3} u+a^{2} b \lambda_{1} \lambda_{3} v+a^{2} b \lambda_{2} \lambda_{3} u+a b^{2} \lambda_{4} \lambda_{1} v \\
& +a^{2} b \lambda_{1} \lambda_{4} u+a^{2} b \lambda_{2} \lambda_{3} v+a b^{2} \lambda_{4} \lambda_{2} u+b^{3} \lambda_{4} \lambda_{2} v .
\end{aligned}
$$

Here, for simplicity, if we take $a=b=1$ then we obtain

$$
\breve{\nabla}_{w}^{*} \breve{\nabla}_{w}^{*} w=\left(0, \lambda_{1}^{2}+\lambda_{2} \lambda_{3}+\lambda_{1} \lambda_{4}+\lambda_{2}^{2}, \lambda_{1}^{2}+\lambda_{2} \lambda_{3}+\lambda_{1} \lambda_{4}+\lambda_{2}^{2}, 0\right),
$$

which yields

$$
w \wedge \breve{\nabla}_{w}^{*} \breve{\nabla}_{w}^{*} w=0
$$

Then we have

$$
w \wedge(C(w, w) \xi+B(w, w) N) \wedge\left(\breve{\nabla}_{w}(C(w, w) \xi+B(w, w) N)\right)=0 .
$$

Thus $C(w, w) \xi+B(w, w) N=0$ or $\breve{\nabla}_{w}(C(w, w) \xi+B(w, w) N)=0$. If $C(w, w) \xi+$ $B(w, w) N=0$, then $C=B=0$, at $p \in N^{\prime}$, which implies that $N^{\prime}$ is totally geodesic and totally umbilical. If $\breve{\nabla}_{w}(C(w, w) \xi+B(w, w) N)=0$, then we have

$$
w(C(w, w)) \xi+w(B(w, w)) N-C(w, w) A_{\xi}^{*} w-B(w, w) A_{N} w=0 .
$$

Hence $C(w, w) A_{\xi}^{*} w+B(w, w) A_{N} w=0$, we find

$$
A_{\xi}^{*} w=-\frac{B(w, w)}{C(w, w)} A_{N} w
$$

at $p \in N^{\prime}$, which shows that $N^{\prime}$ is a screen conformal lightlike hypersurface. 
Consequently, we have the following.

Theorem 2. Let $N$ be a lightlike hypersurface of $\mathbb{R}_{1}^{4}$. Then $N$ has non-degenerate planar normal sections if and only if either $N$ is totally umbilical and screen conformal or $N$ is totally geodesic.

Proof. Assume that $N$ is a totally umbilical and screen conformal lightlike hypersurface of $\mathbb{R}_{1}^{4}$. Then we have $A_{\xi}^{*} w=\beta w, \beta \in \mathbb{R}$. By using (25), 27) and 29], we obtain

$$
\alpha^{\prime \prime \prime}(s) \wedge \alpha^{\prime \prime}(s) \wedge \alpha^{\prime}(s)=0 .
$$

If we consider that $N$ is totally geodesic, then, we have $C=B=0$ and from 36 (38), we see that $w, \breve{\nabla}_{w}^{*} w$ and $\breve{\nabla}_{w}^{*} \breve{\nabla}_{w}^{*} w$ belong to $S\left(T N^{\prime}\right)$. Since $\operatorname{dim}\left(S\left(T N^{\prime}\right)\right)=2$, we conclude that $\alpha^{\prime}, \alpha^{\prime \prime}, \alpha^{\prime \prime \prime}$ are linearly dependent.

Conversely, we assume that $N$ has non-degenerate planar normal sections. Then, from 42- 44 we complete the proof.

Theorem 3. Let $(N, g, S(T N))$ be a screen conformal non-umbilical lightlike hypersurface of $\mathbb{R}_{1}^{4}$. Then, for $T(w, w)=C(w, w) \xi+B(w, w) N$, the following statements are equivalent:

(1) $\left(\breve{\nabla}_{w} T\right)(w, w)=0$, for every spacelike vector $w \in S(T N)$,

(2) $\breve{\nabla} T=0$,

(3) $N$ has non-degenerate planar normal sections and each normal section at $p$ has one of its vertices at $p$.

Note that, by the vertex of curve $\alpha(s)$ we mean a point $p$ on $\alpha$ such that its curvature $\kappa$ satisfies $\frac{d \kappa^{2}(p)}{d s}=0$, where $\kappa^{2}=\left\langle\alpha^{\prime \prime}(s), \alpha^{\prime \prime}(s)\right\rangle$.

Proof. From (25), 26), we have

$$
\left(\breve{\nabla}_{w} T\right)(w, w)=\breve{\nabla}_{w} T(w, w),
$$

which shows $\left(\breve{\nabla}_{w} T\right)(w, w)=0$ if and only if $\breve{\nabla} T=0$.

Assume that $\breve{\nabla} T=0$. Then $N^{\prime}$ is totally geodesic and Theorem 2 implies that $\hat{N}$ has (pointwise) planar normal sections. Let the $\alpha(s)$ be a normal section of $N$ at $p$ in a given direction $w \in S\left(T N^{\prime}\right)$. Then 25 shows that the curvature $\kappa(s)$ of $\alpha(s)$ satisfies

$$
\begin{aligned}
\kappa^{2}(s) & =\left\langle\alpha^{\prime \prime}(s), \alpha^{\prime \prime}(s)\right\rangle \\
& =2 C(w, w) B(w, w) \\
& =\langle T(w, w), T(w, w)\rangle
\end{aligned}
$$

where $w=\alpha^{\prime}(s)$. Therefore we find

$$
\frac{d \kappa^{2}(p)}{d s}=\left\langle\breve{\nabla}_{w} T(w, w), T(w, w)\right\rangle=\left\langle\left(\breve{\nabla}_{w} T\right)(w, w), T(w, w)\right\rangle .
$$


Since $\breve{\nabla}_{w} T(w, w)=0$, this implies

$$
\frac{d \kappa^{2}(0)}{d s}=0,
$$

at $p=\alpha(0)$. Thus $p$ is a vertex of the normal section $\alpha(s)$.

If $N^{\prime}$ has planar normal sections, then by using Theorem 2 we have

$$
T(w, w) \wedge\left(\breve{\nabla}_{w} T\right)(w, w)=0 .
$$

If $p$ is a vertex of $\alpha(s)$, then we have

$$
\frac{d \kappa^{2}(0)}{d s}=0
$$

Thus, since $N$ has planar normal sections, using 46 we find

$$
\begin{aligned}
\alpha^{\prime}(s) \wedge \alpha^{\prime \prime}(s) \wedge \alpha^{\prime \prime \prime}(s)= & w \wedge\left(\breve{\nabla}_{w}^{*} w+T(w, w)\right) \\
& \wedge\left(\breve{\nabla}_{w}^{*} \breve{\nabla}_{w}^{*} w+t T(w, w)+\left(\breve{\nabla}_{w} T\right)(w, w)\right)=0,
\end{aligned}
$$

which yields

$$
T(w, w) \wedge\left(\breve{\nabla}_{w} T\right)(w, w)=0
$$

and

$$
\left\langle\left(\breve{\nabla}_{w} T\right)(w, w), T(w, w)\right\rangle=0 .
$$

Combining 47) and (48) we obtain either $\left(\breve{\nabla}_{w} T\right)(w, w)=0$ or $T(w, w)=0$. Let us define $U=\left\{w \in S\left(T N^{\prime}\right) \mid T(w, w)=0\right\}$. If $\operatorname{int}(U) \neq \varnothing$, we obtain $\left(\breve{\nabla}_{w} T\right)(w, w)=$ 0 on $\operatorname{int}(U)$. Thus, by continuity we have $\breve{\nabla} T=0$.

Considering those obtained results above with [12], we give the following example.

Example 4. Let $\mathbb{R}_{1}^{4}$ be the space $\mathbb{R}^{4}$ endowed with the semi-Euclidean metric

$$
\breve{g}(x, y)=-u_{0} v_{0}+\sum_{a=1}^{3} u_{a} v_{a}, \quad u=\sum_{a=0}^{3} u_{a} \frac{\partial}{\partial u_{a}} .
$$

Consider the null cone of $\mathbb{R}_{1}^{4}$ given by

$$
\wedge_{0}^{3}=\left\{\left(u_{0}, u_{1}, u_{2}, u_{3}\right) \mid-u_{0}^{2}+u_{1}^{2}+u_{2}^{2}+u_{3}^{2}=0, u_{0}, u_{1}, u_{2}, u_{3} \in \mathbb{R}\right\} .
$$

The radical bundle of null cone is

$$
\xi=u_{0} \frac{\partial}{\partial u_{0}}+u_{1} \frac{\partial}{\partial u_{1}}+u_{2} \frac{\partial}{\partial u_{2}}+u_{3} \frac{\partial}{\partial u_{3}}
$$

and screen distribution is spanned by

$$
w=-u_{2} \frac{\partial}{\partial u_{1}}+u_{1} \frac{\partial}{\partial u_{2}}-u_{3} \frac{\partial}{\partial u_{3}} .
$$


Then the lightlike transversal vector bundle is given by

$$
\operatorname{Itr}\left(T \wedge_{0}^{3}\right)=\operatorname{Span}\left\{N=\frac{1}{2\left(u_{0}\right)^{2}}\left(-u_{0} \frac{\partial}{\partial u_{0}}+u_{1} \frac{\partial}{\partial u_{1}}+u_{2} \frac{\partial}{\partial u_{2}}+u_{3} \frac{\partial}{\partial u_{3}}\right)\right\} .
$$

Let $\wedge_{0}^{3}$ be a lightlike hypersurfaces of $\mathbb{R}_{1}^{4}$. For a point $p$ in $\wedge_{0}^{3}$ and a lightlike vector $\xi$ which spans the radical distribution of a lightlike hypersurface, the vector $\xi$ and transversal space $\operatorname{tr}\left(T \wedge_{0}^{3}\right)$ to $\wedge_{0}^{3}$ at $p$ determine a 2 - dimensional subspace $E(p, \xi)$ in $\mathbb{R}_{1}^{4}$ through $p$. The intersection of $\wedge_{0}^{3}$ and $E(p, \xi)$ gives a lightlike curve $\alpha$ in a neighborhood of $p$, which is called the normal section of $\wedge_{0}^{3}$ at the point $p$ in the direction of $\xi$. Therefore, we have

$$
\begin{aligned}
\breve{\nabla}_{\xi} \xi & =u_{0} \frac{\partial}{\partial u_{0}}+u_{1} \frac{\partial}{\partial u_{1}}+u_{2} \frac{\partial}{\partial u_{2}}+u_{3} \frac{\partial}{\partial u_{3}} \\
\breve{\nabla}_{\xi} \breve{\nabla}_{\xi} \xi & =u_{0} \frac{\partial}{\partial u_{0}}+u_{1} \frac{\partial}{\partial u_{1}}+u_{2} \frac{\partial}{\partial u_{2}}+u_{3} \frac{\partial}{\partial u_{3}} .
\end{aligned}
$$

Then, we obtain

$$
\alpha^{\prime \prime \prime}(s) \wedge \alpha^{\prime \prime}(s) \wedge \alpha^{\prime}(s)=0
$$

which shows that null cone has degenerate planar normal sections.

On the other hand, by direct computations, we find

$$
\breve{\nabla}_{\breve{\xi}} w=\breve{\nabla}_{\xi} w=w
$$

and

$$
A_{N} w=\frac{1}{2\left(u_{0}\right)^{2}} A_{\xi}^{*} w .
$$

Namely, $\wedge_{0}^{3}$ is a screen conformal lightlike hypersurface of $\mathbb{R}_{1}^{4}[\underline{5}$.

Now, for a point $p$ in $\wedge_{0}^{3}$ and a non-degenerate vector $w$ tangent to $\wedge_{0}^{3}$ at $p$ $\left(w \in S\left(T \wedge_{0}^{3}\right)\right)$, the vector $w$ and transversal space $\operatorname{tr}\left(T \wedge_{0}^{3}\right)$ to $N$ at $p$ determine a 2- dimensional subspace $E(p, w)$ in $\mathbb{R}_{1}^{4}$ through $p$. The intersection of $\wedge_{0}^{3}$ and $E(p, w)$ gives a non-degenerate curve $\alpha$ in a neighborhood of $p$. Therefore, we have

$$
\begin{aligned}
\alpha^{\prime} & =w=-u_{2} \frac{\partial}{\partial u_{1}}+u_{1} \frac{\partial}{\partial u_{2}}-u_{3} \frac{\partial}{\partial u_{3}}, \\
\alpha^{\prime \prime} & =\breve{\nabla}_{w} w+B(w, w) N \\
& =\frac{1}{2} u_{0} \frac{\partial}{\partial u_{0}}-\frac{3}{2} u_{1} \frac{\partial}{\partial u_{1}}-\frac{3}{2} u_{2} \frac{\partial}{\partial u_{2}}-\frac{3}{2} u_{3} \frac{\partial}{\partial u_{3}}, \\
\alpha^{\prime \prime \prime} & =\breve{\nabla}_{w} \breve{\nabla}_{w} w+w(B(w, w)) N+B(w, w) \breve{\nabla}_{w} N \\
& =\breve{\nabla}_{w} \breve{\nabla}_{w} w+B\left(w, \breve{\nabla}_{w} w\right) N+w(B(w, w)) N-B(w, w) A_{N} w .
\end{aligned}
$$

Using $A_{N} w$ in $\alpha^{\prime \prime \prime}$ we find

$$
\alpha^{\prime \prime \prime}=-\frac{1}{2}\left(-u_{2} \frac{\partial}{\partial u_{1}}+u_{1} \frac{\partial}{\partial u_{2}}-u_{3} \frac{\partial}{\partial u_{3}}\right) .
$$


Therefore $\alpha^{\prime \prime \prime}$ and $\alpha^{\prime}$ are linearly dependent at $p \in \wedge_{0}^{3}$ and we have

$$
\alpha^{\prime} \wedge \alpha^{\prime \prime} \wedge \alpha^{\prime \prime \prime}=0 \text {. }
$$

Namely, $\wedge_{0}^{3}$ has non-degenerate planar normal sections.

\section{REFERENCES}

[1] Atindogbe, C. and Duggal, K. L., Conformal screen on lightlike hypersurfaces, Int. J. Pure Appl. Math. 11(4), (2004), 421-442.

[2] Chen, B. Y., Classification of hypersurfaces with Planar Normal Sections, Journal of Geometry, Vol.20 (1983), 122-127.

[3] Duggal, K. L. and Bejancu, A., Lightlike hypersurfaces of Semi-Riemannian Manifolds and Applications, Kluwer Academic Publisher, 1996.

[4] Duggal, K. L. and Jin, D. H., Null Curves and hypersurfaces of Semi Riemannian Manifolds, World Scientific, 2007.

[5] Duggal, K. L. and Şahin B., Differential Geometry of Lightlike hypersurfaces, Springer Birkhauser, 2010.

[6] Kim, Y. H., Minimal hypersurfaces of pseudo-Euclidean spaces with geodesic normal sections, Differential Geometry and its Applications, 5 (1995), 321-329.

[7] Kim, Y. H., Pseudo-Riemannian hypersurfaces with pointwise planar normal sections, Math. J. Okayama Univ., 34 (1992), 249-257.

[8] Kim, Y. H., Hypersurfaces in a pseudo-Euclidean space with planar normal sections, Journal of Geometry, vol.35 (1989), 120-131.

[9] Li. S. J., Hypersurfaces with Pointwise Planar Normal Sections in a sphere, Journal of Geometry, 70 (2001), 101-107.

[10] O’Neill, B., Semi Riemannian Geometry with Applications to Relativity, Academic Press, 1983.

[11] Erdoğan, F. E., Güneş, R. and Şahin, B., Half-lightlike hypersurface with planar normal sections in $\mathrm{R}_{2}^{4}$, Turk. J. Math. Vol.38 (2014), 764-777.

[12] Erdoğan, F. E., Şahin, B. and Güneş, R., Lightlike hypersurfaces with planar normal section in Minkowski 3-space, Int. Electron. J. Geom., Vol.7, no.1, 2014.

[13] Erdoğan, F. E., Yüksel Perktaş, S. and Güneş, R. , Half Lightlike hypersurfaces with degenerate and non-degenerate planar normal sections in pseudo-Euclidean spaces, International Journal of Geometry, Vol. 7, No.1 (2018), 37-53.

Current address: Faculty of Science, Department of Mathematics, Ege University, İzmir, TURKEY

E-mail address: feyza.esra.erdogan@ege.edu.tr

Current address: Faculty of Art and Science, Department of Mathematics, Adiyaman University Adiyaman, TURKEY

E-mail address: sperktas@adiyaman.edu.tr

ORCID Address: http://orcid.org/0000-0002-8848-0621 